\title{
$\beta$-asarone suppresses HCT116 colon cancer cell proliferation and liver metastasis in part by activating the innate immune system
}

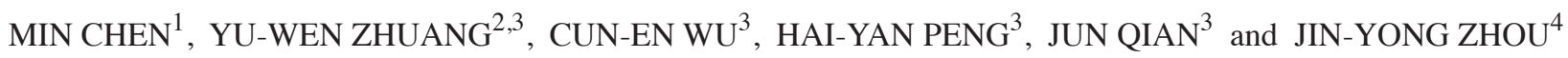 \\ ${ }^{1}$ General Internal Medicine Department, Jiangsu Province Hospital of Chinese Medicine, \\ Affiliated Hospital of Nanjing University of Chinese Medicine, Nanjing, Jiangsu 210029; \\ ${ }^{2}$ Traditional Chinese Medicine Department, Jinling Hospital, School of Medicine, Nanjing University, Nanjing, \\ Jiangsu 210002; ${ }^{3}$ Oncology Department and ${ }^{4}$ Central Laboratory, Jiangsu Province Hospital of Chinese Medicine, \\ Affiliated Hospital of Nanjing University of Chinese Medicine, Nanjing, Jiangsu 210029, P.R. China
}

Received October 27, 2020; Accepted March 11, 2021

DOI: $10.3892 / \mathrm{ol} .2021 .12696$

\begin{abstract}
Studies have revealed that $\beta$-asarone exerts a powerful inhibitory effect on the proliferation of human cancer cells. The authors' previous study demonstrated that $\beta$-asarone could induce LoVo colon cancer cell apoptosis in vitro and in vivo, indicating its anticancer properties. The present study aimed to determine the antineoplastic effect of $\beta$-asarone in HCT116 colon cancer cells. An in vitro proliferation assay using a real time cell analyzer demonstrated that $\beta$-asarone effectively decreased HCT116 cell proliferation in a dose-dependent manner. Bioinformatics analysis revealed that differentially expressed genes following $\beta$-asarone inhibition were involved in the 'cell cycle', 'cell division', 'cell proliferation' and 'apoptosis'. Subsequently, a xenograft assay evidenced the inhibitory effect of $\beta$-asarone on the growth of HCT116 tumors in vivo. Further detection of immune-associated cytokines and cells suggested that $\beta$-asarone might be involved in the antitumor immune response by stimulating granulocyte-colony stimulating factor and increasing the number of macrophage cells in the spleen. Additionally, a murine model of splenic-transplantation verified the strong suppressive role of $\beta$-asarone in colon cancer liver metastasis in vivo. Taken together, the results of the current study revealed that $\beta$-asarone decreased HCT116 colon cancer cell proliferation and liver metastasis
\end{abstract}

Correspondence to: Dr Jin-Yong Zhou, Central Laboratory, Jiangsu Province Hospital of Chinese Medicine, Affiliated Hospital of Nanjing University of Chinese Medicine, 155 Hanzhong Road, Nanjing, Jiangsu 210029, P.R. China

E-mail: jinyongzhou@njucm.edu.cn

Professor Jun Qian, Oncology Department, Jiangsu Province Hospital of Chinese Medicine, Affiliated Hospital of Nanjing University of Chinese Medicine, 155 Hanzhong Road, Nanjing, Jiangsu 210029, P.R. China

E-mail: yfy0101@njucm.edu.cn

Key words: $\beta$-asarone, colon cancer, proliferation, liver metastasis, immune system potentially by activating the innate immune system, supporting the multi-system regulation theory and providing a basis for further mechanistic studies on colon cancer.

\section{Introduction}

Epidemiological statistics have revealed that the morbidity and mortality of patients with colon cancer are both ranked third in the world of all cancer types (1). Numerous Chinese patients are diagnosed with advanced colon carcinoma due to not receiving colonoscopy and a lack of obvious early symptoms (2). A large number of patients initially visit their doctor due to the occurrence of blood in their stool, along with symptoms of stomach pain and a change in bowel movements (2). Although surgical therapy is widely performed, the 5-year relative survival rate of patients with colon cancer was $63.2 \%$, and the overall survival rate was 52\% in the Swiss population between 1996 and 2008 (3). It is well-known that aberrant cell proliferation induces tumor initiation, with subsequent metastasis aggravating the deterioration of patients with cancer. Metastasis has been recognized as the most fatal feature of advanced malignancy, leading to $66.7 \%$ of deaths caused by solid tumors according to population-based data in Norway between 2005 and 2015 (4).

Over the past few decades, chemotherapy has been considered one of the most effective therapies for colon cancer except for surgery. Conventionally in China, 5-fluorouracil (5-Fu) alone or in combination with other anticancer agents (such as irinotecan or oxaliplatin) has been frequently administered and has been shown to improve the survival rate of patients with partial colon cancer $(5,6)$. In addition, the anticarcinogenic effect of several traditional Chinese medicines has also been emphasized in colon cancer $(7,8)$. The authors' previous study demonstrated that modified Si-Jun-Zi Decoction (SJZ), a Chinese medicinal formula, could inhibit colon cancer liver metastasis by increasing the content of macrophage cells (9). A second Chinese medicine, acorus calamus, or its bioactive phytochemical $\beta$-asarone, has been widely reported to possess antitumor and chemopreventive activities in multiple carcinomas, including lung cancer (10), gastric cancer (11) and glioma (12). The suppressive role of $\beta$-asarone in gastric cancer cell proliferation has also been indicated (13). A previous study demonstrated that $\beta$-asarone 
could induce LoVo colon cancer cell apoptosis in vitro and in vivo, suggesting its anticancer properties in colon cancer (14). Furthermore, the study determined that LoVo cell proliferation was reliant on $\beta$-asarone in a time and dose-dependent manner. Moreover, apoptosis could be induced through the mitochondrial/caspase pathway in vitro. Nude mice xenografts with LoVo cells have previously been established to demonstrate growth-suppressing and apoptosis-promoting $\beta$-asarone activity in vivo (14). However, the functional role of $\beta$-asarone on HCT116 colon cancer cells in vitro and in vivo has not yet been elucidated.

The current study aimed to identify the antineoplastic effect of $\beta$-asarone in HCT116 colon cancer cells using in vitro and in vivo experiments. Nude mice are a group of mutant mice with a T lymphocyte deficiency, but with a healthy and undamaged system of innate immunocyte, including macrophage cells, neutrophile granulocytes and natural killer cells $(15,16)$. Therefore, the aim of the study was to focus on the effect of $\beta$-asarone on the innate immune system and perform xenograft tumor and intrasplenic transplantation assays in nude mice. Taken together, the results of the current study further revealed the anticancer effects of $\beta$-asarone in HCT116 colon cancer cells, supporting the possibility of its multi-system regulation, as well as providing supporting information for future $\beta$-asarone-associated research using LoVo colon cancer cells.

\section{Materials and methods}

Chemicals and preparation. $\beta$-asarone was purchased from Sigma-Aldrich (Merck KGaA; cat. no. 221074; batch no. STBF1732V; purity, 96.7\%) and dissolved in DMSO (Beyotime Institute of Biotechnology) for in vitro assays and edible oil solvent for in vivo assays. In particular, $\beta$-asarone dissolved in edible oil was made into concentrations of 50 or $100 \mathrm{mg} / \mathrm{kg}$ body weight, and used for intragastric administration in nude mice. Control mice received the same volume of normal saline by intragastric administration. $\alpha$-asarone and $\beta$-asarone are isomerides (17). $\alpha$-asarone was also used in the xenograft tumor assay and the intrasplenic implantation model to distinguish if different structures could affect the inhibitory effect of the drug.

Cell culture and cell proliferation assay. The human colon cancer cell line, HCT116, was purchased from The Type Culture Collection of the Chinese Academy of Sciences and cultured in RPMI-1640 medium supplemented with $10 \%$ bovine serum (both Biological Industries), penicillin (100 U/ml) and streptomycin $(100 \mu \mathrm{g} / \mathrm{ml})$ in a water-saturated atmosphere at $37^{\circ} \mathrm{C}$ with $5 \% \mathrm{CO}_{2}$. The Real-Time Cell Analyzer (RTCA; ACEA Bioscience, Inc.; Agilent) was used to determine the effect of $\beta$-asarone on HCT116 cell proliferation according to the instruction manual. HCT-116 cells $(\sim 5,000)$ were seeded in each well of an e-plate (ACEA Bioscience, Inc.; Agilent) and incubated at $37^{\circ} \mathrm{C}$ with $5 \% \mathrm{CO}_{2}$. After $24 \mathrm{~h}$, two-fold serial dilutions of $\beta$-asarone ranging from 500, 250, 125, 62.5 and $31 \mu \mathrm{mol} / 1$ were added to the wells accompanied with blank and DMSO controls. RTCA Software 2.0 (ACEA Bioscience, Inc.; Agilent) was used to evaluate the Cell Index (CI) value, which reflected the cell proliferation profile based on impedance measurement. The CI values were normalized at the time of treatment and continuously monitored for $>72 \mathrm{~h}$.
Gene set enrichment analysis. We obtained the RNA transcriptome sequencing results from LC Sciences LLC (https://www.lcsciences.com/), which offers a sequencing service. The differentially expressed genes (DEGs) identified following $\beta$-asarone treatment for 24 and $48 \mathrm{~h}$ were analyzed via pathway analysis [including Gene Ontology (GO) and Kyoto Encyclopedia of Genes and Genomes (KEGG)] using the edge R package. (http://www.bioconductor.org/packages/release/bioc/html/edgeR.html) implemented in $\mathrm{R}$ version 4.0.1 (18). Analysis was performed based on the Wallenius non-central hyper-geometric distribution.

Animals and ethics statement. In total, 88 male Balb/c $\mathrm{nu} / \mathrm{nu}$ mice aged 4-6 weeks with an average weight of $\sim 22 \mathrm{~g}$ were purchased from the Comparative Medicine Centre of Yangzhou University (animal certificate no. 0038475). Mice were maintained under specific pathogen-free conditions at $25^{\circ} \mathrm{C}$ with a $12 \mathrm{~h}$ light-dark cycle. Food and water were available ad libitum. All experimental procedures were approved by The Animal Ethics Committee of the Affiliated Hospital of Nanjing University of Chinese Medicine (Nanjing, China; approval no. 2018 DW-01-03). Experiments were conducted after a 1-week acclimatization period. For surgical anesthesia, $120 \mathrm{mg} / \mathrm{kg}$ ketamine and $10 \mathrm{mg} / \mathrm{kg}$ xylazine were applied intraperitoneally. For euthanasia, an intraperitoneal injection of $200 \mathrm{mg} / \mathrm{kg}$ sodium pentobarbital (overdose) was administered. After confirming the cessation of murine heartbeats, the tumors were collected for further analysis. The humane endpoints will come when either the maximum diameter of tumor is larger than $2.0 \mathrm{~cm}$ or the loss is more than $20 \%$ of the beginning body weight.

Animal groups and treatment in the xenograft tumor assay. HCT116 cancer cells (2 million) were suspended with PBS and subcutaneously transplanted into the right posterior flank of every mouse of 60 nude mice, which were then randomly divided into the following four groups ( $\mathrm{n}=10 \mathrm{mice} / \mathrm{group}$ ): i) Negative control group; ii) $\beta$-asarone $50 \mathrm{mg} / \mathrm{kg}$ group; iii) $\beta$-asarone $100 \mathrm{mg} / \mathrm{kg}$ group; and iv) $\alpha$-asarone $50 \mathrm{mg} / \mathrm{kg}$ group; v) $\alpha$-asarone $100 \mathrm{mg} / \mathrm{kg}$ group and vi) 5 -Fu $25 \mathrm{mg} / \mathrm{kg}$ group. All treatments were administered from the 3rd day after injection of HCT116 cells. Tumor growth was examined every 4 days, and tumor volumes were calculated using the following equation: Volume $=0.5 \mathrm{x}$ longitudinal diameter $\mathrm{x}$ latitudinal diameter ${ }^{2}$. At 4 weeks after treatment, mice were euthanized as aforementioned, subcutaneous tumors were imaged and tumor weights were measured.

Plasma collection for cytokine analysis. Following euthanasia, the peripheral blood of mice was collected. Blood samples in each mouse were aliquoted for cytokine and flow cytometry analysis. Half the plasma obtained from a single mouse within the same group was used to analyze plasma cytokines using the Mouse Cytokine Array Q5 kit (cat. no. QAM-CYT-5-1; Raybiotech, Inc.) as previously described (19).

Flow cytometry analysis of macrophages and neutrophil granulocytes. In addition to samples used in cytokines analysis, the remaining blood samples obtained from each mouse were used for the flow cytometry analysis of macrophages and 
neutrophil granulocytes. Additionally, spleen and liver cells were obtained from each mouse after sacrifice and used for flow cytometric analysis. Accuri ${ }^{\mathrm{TM}} \mathrm{C} 6$ flow cytometry and BD Accuri ${ }^{\mathrm{TM}}$ C6 software (version 1.0.264.21) from Becton, Dickinson and Company were used for above flow cytometry analysis. For cell surface marker staining, splenocytes and liver cells from mice were cut into cell suspensions. The cell suspensions were sequentially filtered through $70-\mu \mathrm{m}$ (cat no. 340635) and 50- $\mu \mathrm{m}$ (cat no. 340592) cell strainers (both BD Biosciences), and the single cells were collected by centrifugation at $300 \mathrm{x} \mathrm{g}$ for $5 \mathrm{~min}$ at $4^{\circ} \mathrm{C}$. After treatment with $5 \mu \mathrm{l} \mathrm{FcR}$ blocking buffer in $100 \mu \mathrm{l}$ reaction system for $15 \mathrm{~min}$ at $4^{\circ} \mathrm{C}$ (cat no. 130-092-575; Miltenyi Biotec, Inc.), the cells were immediately prepared for staining. All the cells were incubated $15 \mathrm{~min}$ at $4^{\circ} \mathrm{C}$ with PE-Vio770-conjugated anti-mouse CD45 mAb (cat no. 130-117-529; Miltenyi Biotec, Inc.) for chosen the leukocytes, followed by stained other antibodies for $30 \mathrm{~min}$ at $4^{\circ} \mathrm{C}$ in the dark. Double positive CD11b-APC (cat no. 17-0112-83) and F4/80-PE mAb (cat no. 12-4801-82) (both eBioscience; Thermo Fisher Scientific, Inc.) staining was for macrophages and double positive CD11b-APC and Ly-6G-FITC mAb (Biolegend, cat no. 127606) staining was for neutrophiles (9).

Intrasplenic transplantation and animal treatment. The fluorescent signals of GFP-HCT116 cells (Nanjing Tran-Medical Inc., http://www.tranmedical.com/xbx.html) were confirmed using flow cytometry. Before intrasplenic implantation, the flow cytometry results showed that $95.9 \%$ cells were GFP positive. GFP-HCT116 cells were diluted with the complete RPMI-1640 medium to a final concentration of $1 \times 10^{8} / \mathrm{ml}$ and intrasplenically transplanted according to the method proposed by Giavazzi et al (20). An intraperitoneal injection of ketamine and xylazine were administered as anesthetic, after which $20 \mu \mathrm{l}$ cell suspension was injected into the spleen of each nude mouse. Animals were subsequently left to recover on a heating pad after surgery and returned to housing cages. Intrasplenically transplanted nude mice $(n=48)$ were then randomly divided into the following four groups: i) Negative control $(n=12)$; ii) 5 -Fu $(n=12)$; iii) $\alpha$-asarone $(n=12)$; and iv) $\beta$-asarone $(n=12)$ groups. The $5-\mathrm{Fu}$ group received a $15 \mathrm{mg} / \mathrm{kg}$ body weight intraperitoneal injection twice a week (on the first day and fourth day). The $\alpha$-asarone and $\beta$-asarone group were administered a $100 \mathrm{mg} / \mathrm{kg}$ body weight intragastric injection once per day. All treatments were applied for a total of 12 consecutive days, followed by an 8-day break of treatment for two cycles. Following sacrifice as aforementioned, animals that did not exhibit a tumor of the spleen or liver were excluded. Of the included mice, the orthotopic splenic tumors were dissected and weighed.

Tumor fluorescence imaging. A fluorescence optical imaging system was used to examine colon cancer liver metastasis in vivo at the day of sacrifice. Mice were euthanized as aforementioned and dissected to observe the liver metastasis of GFP-HCT116 cells. Fluorescent images were acquired using a fluorescence stereo-microscope (model, NSZ-608T; Nanjing Jiangnan Novel Optics Co., Ltd.) equipped with a D510 long-pass emission filter (Chroma Technology Corporation) and a cooled color charge-coupled device camera (Teledyne Technologies Inc.). Image Pro plus 6.0 software (Media
Cybernetics, Inc.) was used to process and analyze fluorescent images. HCT116 cells were labeled with GFP, which indicated that green fluorescence on the liver represented metastatic HCT116 cells.

Statistical analysis. Data are presented as the mean \pm SD. One-way ANOVA, unpaired t-tests or Fisher's exact tests were used to analyze the differences between groups. Dunnett's post hoc tests were used following one-way ANOVA. $\mathrm{P}<0.05$ was considered to indicate a statistically significant difference.

\section{Results}

$\beta$-asarone significantly inhibits the proliferation of HCT116 colon cancer cells. To determine the biological effect of $\beta$-asarone in HCT116 cell malignant behavior, HCT116 cell proliferation was assessed after $\beta$-asarone treatment. As presented in Fig. 1B, human HCT116 colon cancer cells were treated with $31,62.5,125,250$ or $500 \mu \mathrm{M} \beta$-asarone. The results indicated that $\beta$-asarone effectively decreased HCT116 cell proliferation in a dose-dependent manner. Moreover, cells administered $500 \mu \mathrm{M} \beta$-asarone exhibited a $50 \%$ reduction in cell proliferation compared with the control group (Fig. 1B). The grouped means of the normalized CI following 24, 48 and $72 \mathrm{~h} \beta$-asarone treatment are presented in Fig. 1C. The results indicated that only $500 \mu \mathrm{M} \beta$-asarone was statistically significant compared with the control.

GO analysis and DEG pathway enrichment following $\beta$-asarone inhibition in HCT116 cells. To investigate the potential target genes and associated pathways involved in HCT116 cell $\beta$-asarone inhibition on an unbiased basis, RNA transcriptome sequencing following $\beta$-asarone treatment for 24 and $48 \mathrm{~h}$ was performed. The results identified 100 DEGs at 24 and $48 \mathrm{~h}$ treatment (fold-change $>2$; $\mathrm{P}<0.05$ ). Among the results obtained at $24 \mathrm{~h}, 66$ genes were significantly upregulated, while 34 genes were downregulated (Fig. S1A). Analogous data were obtained following 48 h treatment; 61 genes were upregulated and 39 genes were downregulated (Fig. S1C). Pathway analysis (Fig. 2) revealed that the 'regulation of transcription', 'cell cycle', 'cell division', 'apoptosis' and 'DNA replication' were prominent pathways involved in the modulatory effects of $\beta$-asarone after treatment for 24 and $48 \mathrm{~h}$, which may be involved in $\beta$-asarone inhibition-mediated HCT116 cell proliferation. The 'IL-17 signaling pathway' was identified as a regulatory pathway involved in $\beta$-asarone treatment for $48 \mathrm{~h}$, but not in treatment for $24 \mathrm{~h}$, indicating that $\beta$-asarone may have only activated the innate immune system in HCT116 cancer cells at a later point in time (Fig. 2D).

$\beta$-asarone significantly inhibits HCT116 cell tumorigenesis in vivo. To further investigate whether $\beta$-asarone inhibited HCT116 cell tumorigenesis in vivo, HCT116 cells were subcutaneously injected into nude mice. As presented in Fig. 3B, tumor growth following $100 \mathrm{mg} / \mathrm{kg} \beta$-asarone and $25 \mathrm{mg} / \mathrm{kg}$ $5-\mathrm{Fu}$ treatment was markedly reduced compared with the control group. The maximum diameter and volume of a single tumor were $19.30 \mathrm{~mm}$ and $1,758.71 \mathrm{~mm}^{3}$, respectively. Furthermore, images revealed that the tumors of the treatment groups were generally smaller than those of the control 
A<smiles>C/C=C/c1cc(OC)c(OC)cc1OC</smiles>

C

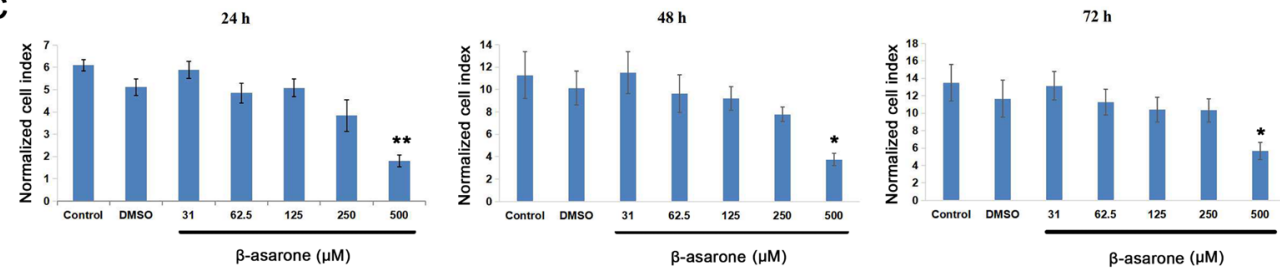

B

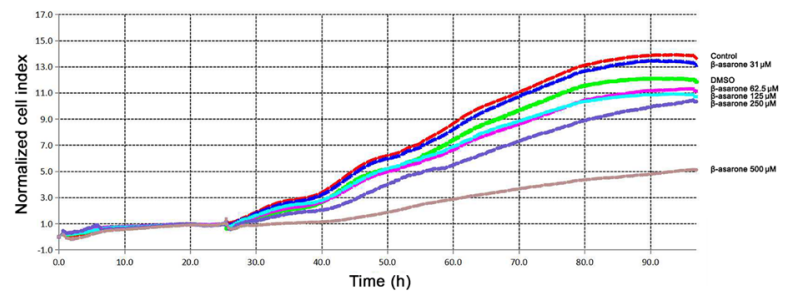

$\beta$-asarone $(\mu \mathrm{M})$

Figure 1. Chemical structure of $\beta$-asarone and cell proliferation assay results. (A) Chemical structure of $\beta$-asarone. (B) $\beta$-asarone effectively repressed HCT116 cell proliferation in a dose-dependent manner. (C) Grouped means of normalized cell indices from 24,48 or $72 \mathrm{~h}$ treatment with $31,62.5,125,250$ or $500 \mu \mathrm{M}$ $\beta$-asarone. "P $\mathrm{P}<0.05$ showed the significant different between $500 \mu \mathrm{M} \beta$-asarone and DMSO after 48 and $72 \mathrm{~h}$ treatment, respectively. ${ }^{* *} \mathrm{P}<0.01$ showed the significant different between $500 \mu \mathrm{M} \beta$-asarone and DMSO after $24 \mathrm{~h}$ treatment.

\section{A}

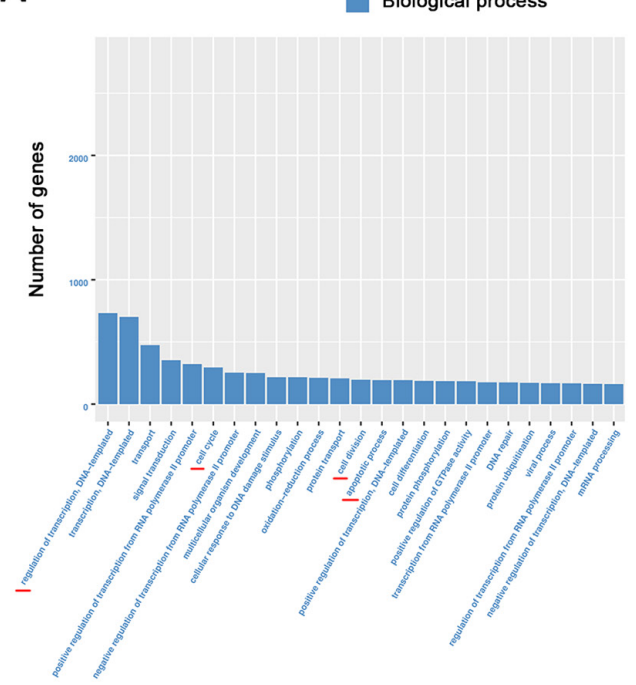

。

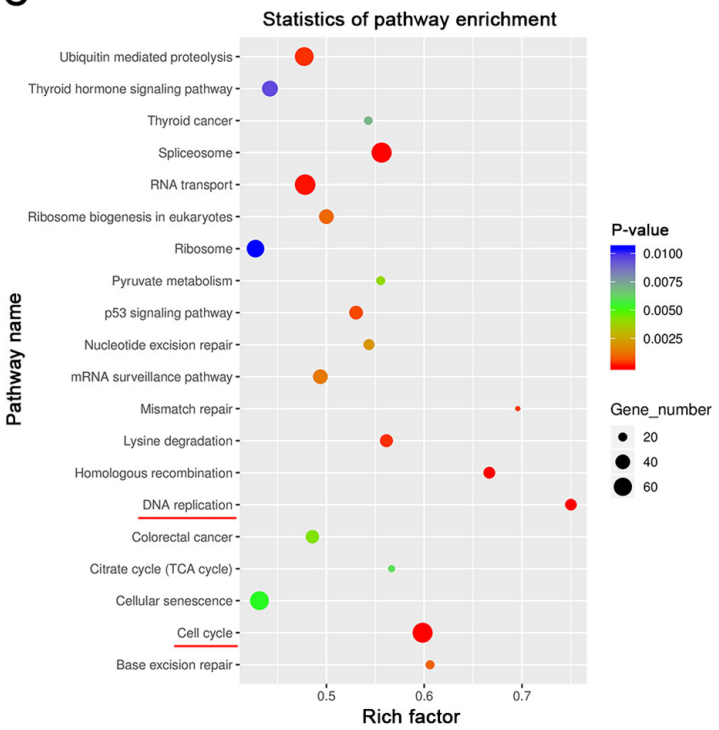

B

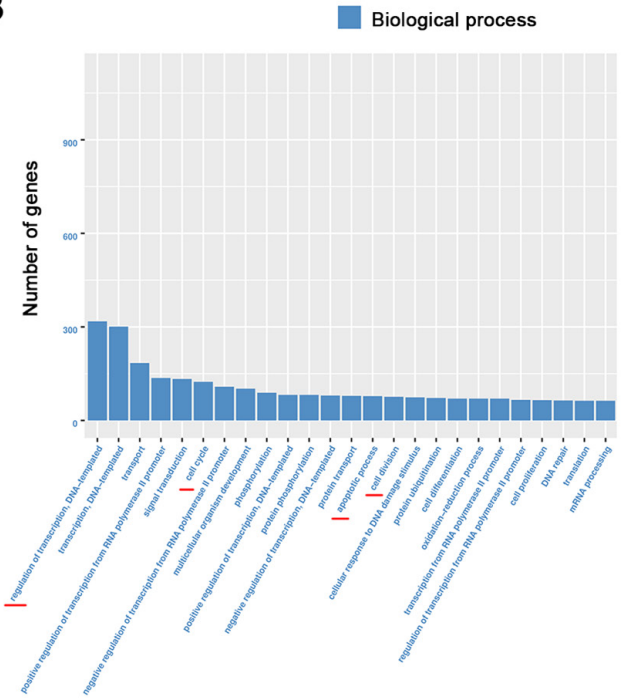

D

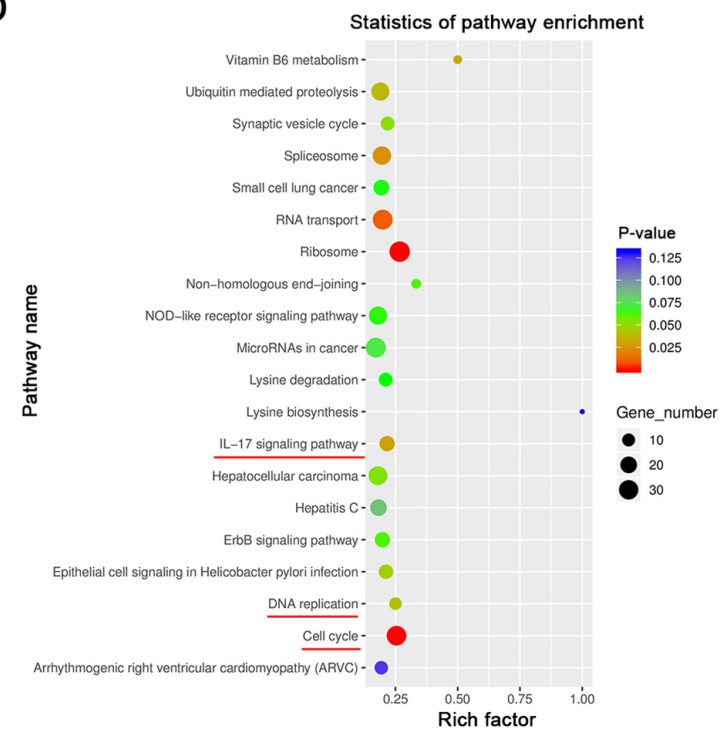

Figure 2. Gene Ontology analysis and pathway enrichment analysis. (A and B) Gene Ontology and (C and D) pathway enrichment analysis for the DEGs at 24 and $48 \mathrm{~h}$ treatment of $\beta$-asarone. DEGs, differentially expressed genes; Num, number. 
A

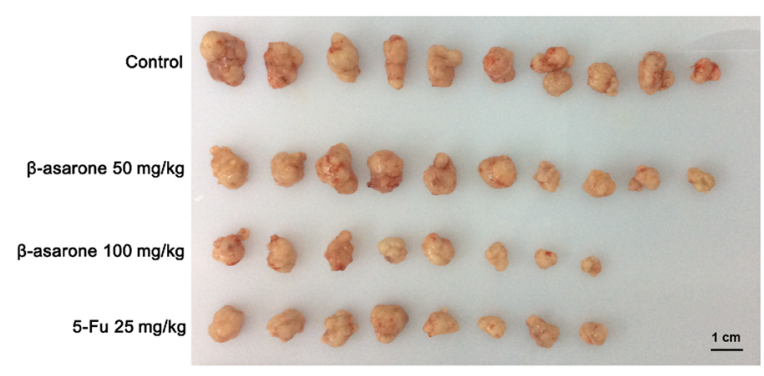

B

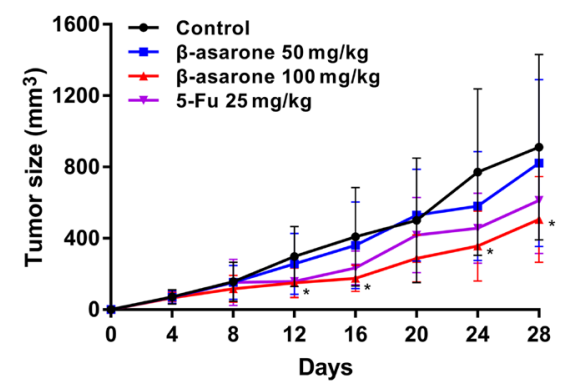

C

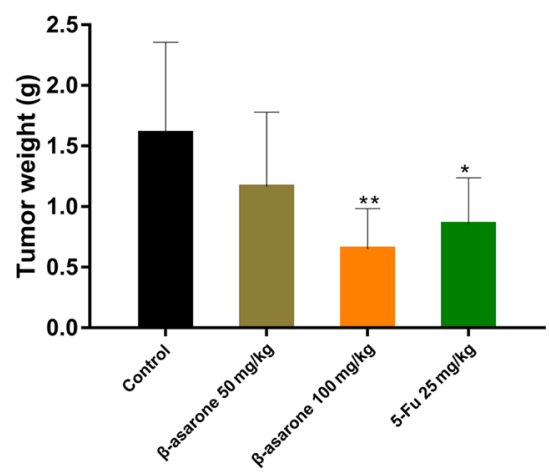

Figure 3. $\beta$-asarone significantly inhibits HCT116 cell tumorigenesis in vivo. (A) Tumors formed in the treated groups were generally smaller compared with those in the control group $(\mathrm{n}=10)$, particularly in those treated with $100 \mathrm{mg} / \mathrm{kg} \beta$-asarone $(\mathrm{n}=8)$. (B) Tumor volumes were measured and calculated every 4 days after $\beta$-asarone treatment. (C) Mean tumor weight of the treatment groups. ${ }^{*} \mathrm{P}<0.05$ and ${ }^{* * *} \mathrm{P}<0.01$ vs. control.

A

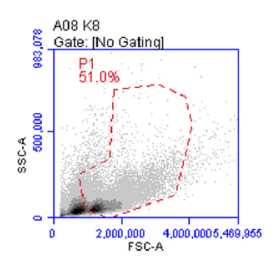

Control

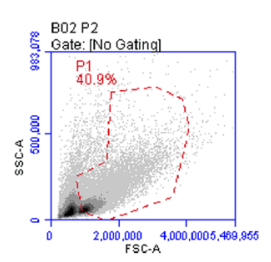

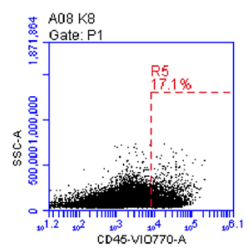
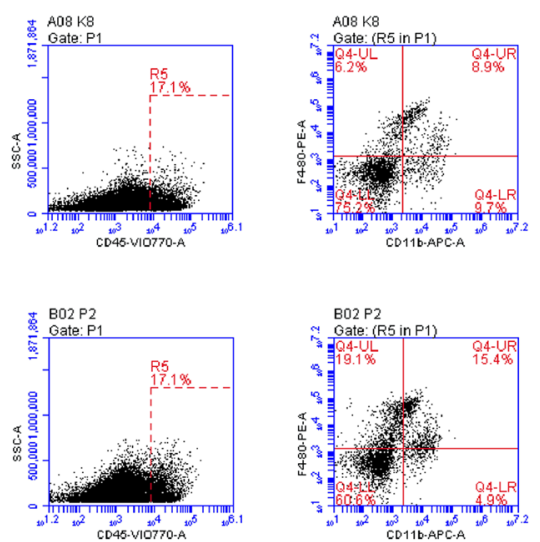

B

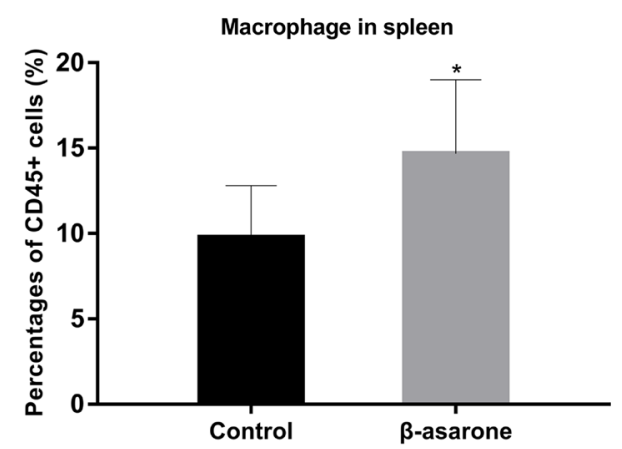

Figure 4. Flow cytometry results of splenic macrophages. (A) Flow cytometry was performed to detect the number of macrophage cells after $\beta$-asarone treatment. P1 gates represent the granulocytes and lymphocytes. Double positive of CD11b-APC and F4/80-PE dots in R5 gate represented the macrophages. (B) Statistical analysis revealed that the percentages of macrophage cells in the spleen increased from $10 \%$ to $15 \%$ after $\beta$-asarone treatment. " $\mathrm{P}<0.05$ vs. control.

group, particularly in mice treated with $100 \mathrm{mg} / \mathrm{kg} \beta$-asarone (Fig. 3A). In addition, the mean tumor weights of the treatment groups were reduced compared with matched control. Moreover, the $100 \mathrm{mg} / \mathrm{kg} \beta$-asarone lavage group exhibited a $50 \%$ reduction in weight compared with the control (Fig. $3 \mathrm{C}$ ). $\alpha$-asarone was also used in xenograft model experiment (Fig. S2A). However, the statistical P-value of tumor weight between control and $\alpha$-asarone groups was $>0.05$, which revealed no statistical significance (Fig. S2B).

$\beta$-asarone may activate the innate immune system of nude mice. The results of cytokine analysis revealed that granulocyte colony-stimulating factor (G-CSF) levels in the peripheral blood of nude mice were significantly increased after $\beta$-asarone treatment (fold-change, $1.78 ; \mathrm{P}=0.02$ ). Additionally, the results of flow cytometry demonstrated that the number of splenic macrophage cells increased by $50 \%$ (from 10 to $15 \%$ ) after $\beta$-asarone treatment (Fig. $4 \mathrm{~A}$ and $\mathrm{B} ; \mathrm{P}<0.05$ ). However, no significant difference in neutrophil granulocyte levels was observed among the treatment groups in murine livers, spleens and peripheral blood (Fig. S3), indicating that the functional role of $\beta$-asarone in tumor suppression may also depend on splenic immune regulation.

Effect of $\beta$-asarone on colon cancer liver metastasis and orthotopic splenic tumors in vivo. Green fluorescence indicated that 
Table I. Effect of different treatments on colon cancer liver metastasis.

\begin{tabular}{lccccr}
\hline Group & $\mathrm{N}^{\mathrm{a}}$ & $\mathrm{N}_{\text {non-metastasis }}$ & $\mathrm{N}_{\text {metastasis }}$ & Rate, $\%$ & OR \\
\hline Negative control & 11 & 1 & 10 & 90.9 & - \\
Positive control $^{\mathrm{b}}$ & 7 & 2 & 5 & 71.4 & 3.68 \\
-asarone $^{\text {-asarone }}$ & 10 & 2 & 8 & 80.0 & 0.528 \\
-asalue & 7 & 5 & 41.7 & 0.586 \\
\hline
\end{tabular}

${ }^{a}$ Those who did not exhibit tumors of the spleen and liver were excluded. ${ }^{\text {b5 }}$-fluorouracil. N, number; OR, odds ratio.

Table II. Effects of different treatments on orthotopic splenic tumor weight in liver metastatic nude mice models.

\begin{tabular}{lcr}
\hline Treatment groups & Tumor weight $^{\mathrm{a}}, \mathrm{g}$ & P-value \\
\hline Negative control & $0.78 \pm 0.66$ & - \\
Positive control $^{\mathrm{c}}$ & $0.51 \pm 0.48$ & 0.573 \\
$\alpha$-asarone & $0.66 \pm 0.59$ & 0.918 \\
$\beta$-asarone & $0.19 \pm 0.12$ & $0.022^{\mathrm{b}}$ \\
\hline
\end{tabular}

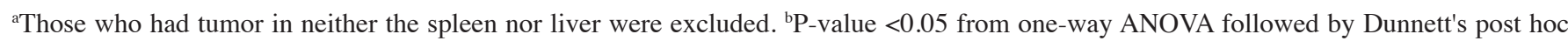
test. ${ }^{\mathrm{c}} 5$-fluorouracil.
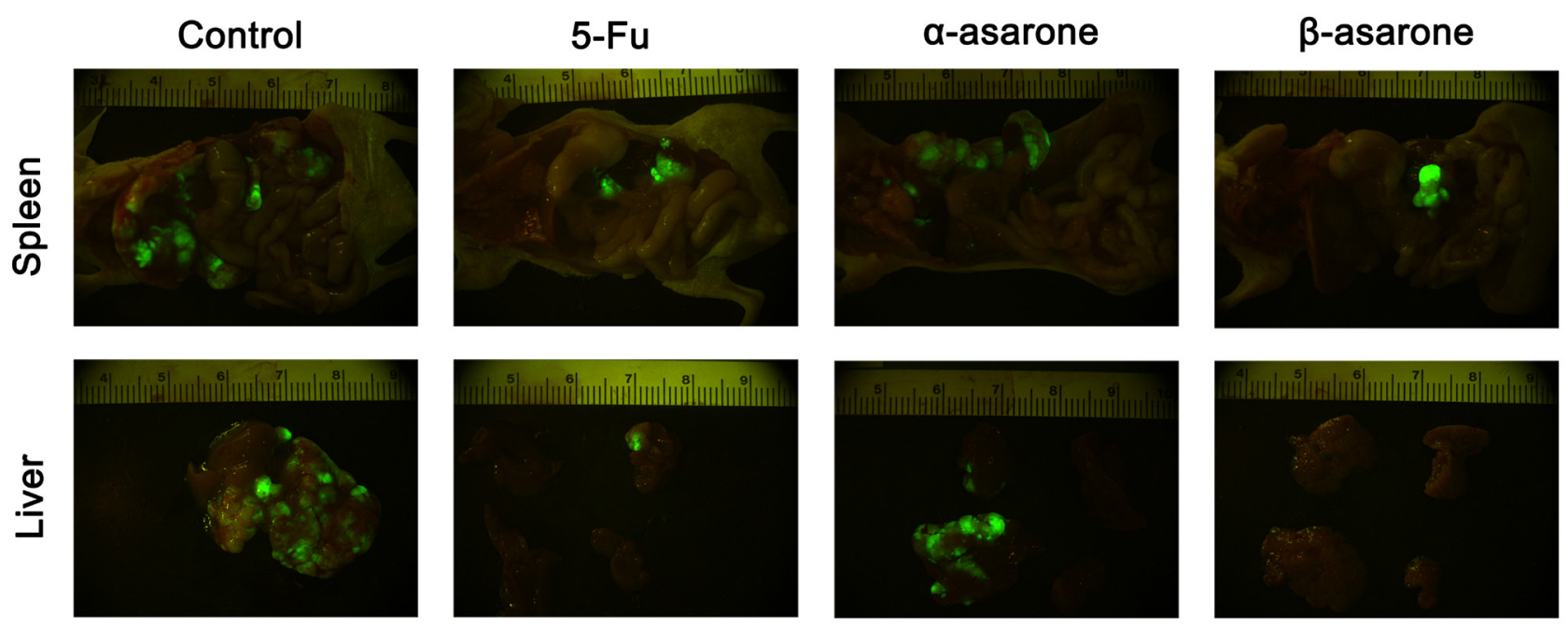

Figure 5. Representative images of colon cancer liver metastasis in different groups. Green fluorescence clusters in the upper images represented orthotopic splenic tumors, whereas the green fluorescence clusters in the lower images represented liver-metastatic tumor tissue.

colon cancer tissues were present. As presented in Fig. 5, the fluorescence of tissue following 5-Fu and $\beta$-asarone treatment was markedly reduced in murine livers compared with the original splenic injection site. Tumor fluorescence images revealed no metastatic tumor tissue in the liver following $\beta$-asarone treatment, indicating the strong inhibitory effect of $\beta$-asarone on colon cancer liver metastasis in vivo. Furthermore, metastatic rate dropped from $90.9 \%$ (10/11 in the control group) to $41.7 \%(5 / 12$ in the $\beta$-asarone-treated group; $\mathrm{P}=0.027)$, suggesting that $\beta$-asarone exerted a strong inhibitory effect on colon cancer liver metastasis in vivo (Table I). Moreover, orthotopic splenic tumor weights were measured, the results of which revealed that the mean tumor weights of the $\beta$-asarone treatment group were significantly decreased compared with the negative control group $(0.19 \pm 0.12 \mathrm{~g}$ vs. $0.78 \pm 0.66 \mathrm{~g}$; $\mathrm{P}=0.022$ ). The results further supported the inhibitory effect of $\beta$-asarone on orthotopic splenic tumors (Table II).

\section{Discussion}

Several conventional Chinese medicines have been commonly used in clinical practice to treat cancer, including shenqi fuzheng, kanglaite, huachansu and cantharidin sodium injections. These treatments have been demonstrated to reduce tumor metastasis and recurrence, and improve the quality of life and survival of patients with hepatocellular carcinoma, non-small cell lung cancer and colorectal cancer (21). Both $\alpha$ - and $\beta$-asarone, the most studied bioactive phytochemicals 
of acorus calamus, have been reported to have multiple pharmacological activities such as antidepressant, antianxiety, anti-Alzheimer's, anti-Parkinson's, antiepileptic, anticancer, antihyperlipidemic, antithrombotic, anticholestatic and radioprotective activities through its interaction with multiple molecular targets (22). $\alpha$-asarone and $\beta$-asarone are isomerides (23). A large number of clinical studies in China have indicated the effectiveness of $\alpha$-asarone against respiratory disorders and epilepsy $(24,25)$; while $\beta$-asarone has been reported to exert biological effects on different human body systems, serving inhibitory functions in numerous human carcinomas $(10,26)$. For instance, $\beta$-asarone exerts antifungal and anthelmintic activity, regulates the nervous system and blocks cholesterol synthesis (27-29). In addition, previous studies have also highlighted the inhibitory function of $\beta$-asarone on tumor metastasis in glioma, lung cancer and gastric cancer cells (10-12). Furthermore, the antiproliferative property of $\beta$-asarone has been reported in gastric cancer and LoVo colon cancer cells in a previous study $(13,14)$. Liu et al $(30)$ revealed that $\beta$-asarone could induce senescence in colorectal cancer cells by increasing the expression of Lamin B1. However, its suppressive function on the proliferation of HCT116 colon cancer cells and subsequent liver metastasis is yet to be fully elucidated.

The current study aimed to determine the antineoplastic role of $\beta$-asarone in HCT116 cells, the results of which revealed that the inhibitory effect of $\beta$-asarone was greater compared with $\alpha$-asarone. The results of the present study demonstrated that $\beta$-asarone effectively repressed HCT116 cell proliferation in a dose-dependent manner. Subsequent GO and pathway analysis revealed that the DEGs identified following $\beta$-asarone inhibition were involved in the 'cell cycle', 'cell division', 'cell proliferation' and 'apoptosis'. Furthermore, xenograft tumor assays indicated the inhibitory role of $\beta$-asarone on HCT116 cell tumorigenesis in vivo. In view of that $\alpha$-asarone and $\beta$-asarone are both isomerides, $\alpha$-asarone was also used in xenograft model experiment to distinguish if different structures could affect the inhibitory effectiveness of the drug. However, there was no statistically significant difference. A nude mouse model of HCT116 cell splenic-transplantation was established to assess liver metastasis, which mimicked the pathogenesis of colon cancer.

Based on ancient Chinese medicinal theory, TCM regulates the human immune system to maintain homeostasis $(31,32)$. Trichosanthin, which is extracted from the Chinese medicinal herb Trichosanthes kirilowi, improves antitumor immunity through the interaction between tumor suppressor in lung cancer 1 and cytotoxic and regulatory T cells (33). Chang and Shen (34) determined that linalool stimulates IFN- $\gamma$, IL-13, IL-2, IL-21, IL-21R, IL-4, IL-6sR and TNF- $\alpha$ secretion, indicating that it exerts cytotoxic effects in the antitumor immunity process. Additionally, our previous study demonstrated that modified SJZ inhibited colon cancer liver metastasis by activating the innate immune system (9). It was similarly demonstrated that plasma GM-CSF and macrophage levels are significantly increased following modified SJZ treatment (9). Nevertheless, the functional role of $\beta$-asarone in colon cancer immunity has not yet been fully elucidated, to the best of our knowledge. The current study assessed the influence of $\beta$-asarone on the innate immune response of HCT116 cells. Cytokine analysis revealed that G-CSF levels in the peripheral blood of nude mice were significantly increased after $\beta$-asarone treatment. G-CSF stimulates the proliferation of neutrophil granulocytes and macrophages $(35,36)$. Neutrophil granulocytes are therefore the major effectors of acute inflammation, as they are one of the first responders during the immune response, migrating to inflammation sites to target bacteria or infiltrating cancer tissue $(37,38)$. An increase in macrophage numbers could also be used to estimate immune system changes. It is well-known that macrophage cells engulf and digest cellular debris, foreign substances and cancer cells, indicating its vital role in non-specific defense (39). Therefore, the current study focused on the innate immune system following $\beta$-asarone treatment and verified $\beta$-asarone's anticancer immunoregulation. However, the downstream target genes of $\beta$-asarone and the potential associated mechanisms remain unclear in HCT116 colon cancer cells, which requires further study.

Previous studies have not provided the evidence for the adverse effect of $\beta$-asarone on normal cells, which could also be a limitation of the present study. Certainly, the possible cytotoxic activity of $\beta$-asarone in normal cells needs to be further explored to improve our understanding of $\beta$-asarone's inhibitory effect on these tumorigenic cells. Additionally, to develop our understanding of tumor growth and metastasis-related biomarkers in tumor tissues/metastases, it would be important to elucidate the mechanisms by which $\beta$-asarone exerts its anti-growth and anti-metastasis activities in vivo. The lack of such investigation is another limitation of the present study, but could be a further area of future research.

In conclusion, the present study demonstrated that $\beta$-asarone exerted an inhibitory effect on the proliferation and metastasis of HCT116 colon cancer cells in vitro and in vivo. In addition, $\beta$-asarone may be involved in the antitumor immune response by stimulating G-CSF and increasing the number of macrophages in the spleen. Collectively, the current data verified the anticancer effects of $\beta$-asarone, both functionally and immunologically, supporting the possibility of its multi-system regulation and providing a basis for future research into colon cancer.

\section{Acknowledgements}

Not applicable.

\section{Funding}

This work was funded by The Priority Academic Program Development of Jiangsu Higher Education Institutions, the National Natural Science Foundation of China (grant no. 81202954), The Natural Science Fund of Jiangsu Province, China (grant no. BK20201499), The Peak Academic Talents Plan in Jiangsu Province Hospital of Chinese Medicine (grant no. k2018yrc25), The Six One Project in Jiangsu Province (grant no. LGY2018062), The State Administration of Traditional Chinese Medicine of the People's Republic of China (National Famous Chinese Medicine Doctor Studio grant no. 2018-119) and The National Traditional Chinese 
Medicine Inheritance and Innovation Platform Construction Project by National Administration of Traditional Chinese Medicine (grant no. 20085-9-5).

\section{Availability of data and materials}

The datasets used and/or analyzed during the current study are available from the corresponding author on reasonable request.

\section{Authors' contributions}

MC and YWZ designed experiments. MC, YWZ and JYZ performed the experiments. MC, CEW, HYP and JQ analyzed the results. CEW and JYZ wrote the manuscript. JQ and JYZ conceived and supervised the project. JYZ and JQ confirm the authenticity of all the raw data. All authors read and approved the final manuscript.

\section{Ethics approval and consent to participate}

All experimental procedures were approved by The Animal Ethics Committee of Affiliated Hospital of Nanjing University of Chinese Medicine (approval no. 2018 DW-01-03) according to the Regulation of Experimental Animal Management (State Scientific and Technological Commission of the People's Republic of China; approval no. 2, 1988) and the Jiangsu Province Experimental Animal Management (Jiangsu province government, China; approval no. 45,2008$)$.

\section{Patient consent for publication}

Not applicable.

\section{Competing interests}

The authors declare that they have no competing interests.

\section{References}

1. Siegel RL, Miller KD, Fuchs HE and Jemal A: Cancer statistics, 2021. CA Cancer J Clin 71: 7-33, 2021.

2. Wu XD, Zeng YY, Wu XJ and Qin HY: The prevalence and correlates of prehospital delay and health belief in chinese patients with colorectal cancer. Gastroenterol Nurs 43: 186-195, 2020.

3. Ukegjini K, Zadnikar M, Warschkow R, Muller S, Schmied BM and Marti L: Baseline mortality-adjusted survival in colon cancer patients. Langenbecks Arch Surg 401: 633-641, 2016.

4. Dillekås H, Rogers MS and Straume O: Are $90 \%$ of deaths from cancer caused by metastases? Cancer Med 8: 5574-5576, 2019.

5. Wu C: Systemic therapy for colon cancer. Surg Oncol Clin N Am 27: 235-242, 2018.

6. Park JH, Zhao M, Han Q, Sun Y, Higuchi T, Sugisawa N, Yamamoto J, Singh SR, Clary B, Bouvet M and Hoffman RM: Efficacy of oral recombinant methioninase combined with oxaliplatinum and 5-fluorouracil on primary colon cancer in a patient-derived orthotopic xenograft mouse model. Biochem Biophys Res Commun 518: 306-310, 2019.

7. Yan S, Yue Y, Wang J, Li W, Sun M, Zeng L and Wang X: Banxia Xiexin decoction, a traditional Chinese medicine, alleviates colon cancer in nude mice. Ann Transl Med 7: 375, 2019.

8. Sun X, Ng TTH, Sham KWY, Zhang L, Chan MTV, Wu WKK and Cheng CHK: Bufalin, a traditional Chinese medicine compound, prevents tumor formation in two murine models of colorectal cancer. Cancer Prev Res (Phila) 12: 653-666, 2019.
9. Zhou JY, Chen M, Wu CE, Zhuang YW, Chen YG and Liu SL: The modified Si-Jun-Zi Decoction attenuates colon cancer liver metastasis by increasing macrophage cells. BMC Complement Altern Med 19: 86, 2019.

10. Wang TL, Ouyang CS and Lin LZ: $\beta$-Asarone suppresses Wnt/ $\beta$-catenin signaling to reduce viability, inhibit migration/invasion/adhesion and induce mitochondria-related apoptosis in lung cancer cells. Biomed Pharmacother 106: 821-830, 2018.

11. Tao H, Ding X, Wu J, Liu S, Sun W, Nie M, Pan X and Zou X: $\beta$-Asarone increases chemosensitivity by inhibiting tumor glycolysis in gastric cancer. Evid Based Complement Alternat Med 2020: 6981520, 2020.

12. Wang N, Han Y, Luo L, Zhang Q, Ning B and Fang Y: $\beta$-Asarone induces cell apoptosis, inhibits cell proliferation and decreases migration and invasion of glioma cells. Biomed Pharmacother 106: 655-664, 2018.

13. Wu J, Zhang XX, Sun QM, Chen M, Liu SL, Zhang X, Zhou JY and Zou X: $\beta$-Asarone inhibits gastric cancer cell proliferation. Oncol Rep 34: 3043-3050, 2015.

14. Zou X, Liu SL, Zhou JY, Wu J, Ling BF and Wang RP: Beta-asarone induces LoVo colon cancer cell apoptosis by up-regulation of caspases through a mitochondrial pathway in vitro and in vivo. Asian Pac J Cancer Prev 13: 5291-5298, 2012.

15. Prehn LM and Outzen HC: Primary tumor immunity in nude mice. Int J Cancer 19: 688-691, 1977.

16. Kenyon RH and Pedersen CE Jr: Immune responses to Rickettsia akari infection in congenitally athymic nude mice. Infect Immun 28: 310-313, 1980.

17. Uebel T, Hermes L, Haupenthal S, Müller L and Esselen M: $\alpha$-Asarone, $\beta$-asarone, and $\gamma$-asarone: Current status of toxicological evaluation. J Appl Toxicol: Nov 25, 2020 (Epub ahead of print).

18. R Core Team: R: A language and environment for statistical computing. R Foundation for Statistical Computing, Vienna, 2012. http://www.R-project.org/.

19. Correnti JM, Cook D, Aksamitiene E, Swarup A, Ogunnaike B, Vadigepalli R and Hoek JB: Adiponectin fine-tuning of liver regeneration dynamics revealed through cellular network modelling. J Physiol 593: 365-383, 2015.

20. Giavazzi R, Garofalo A, Damia G, Garattini S and D'Incalci M: Response to flavone acetic acid (NSC 347512) of primary and metastatic human colorectal carcinoma xenografts. Br J Cancer 57: 277-280, 1988.

21. Qi F, Zhao L, Zhou A, Zhang B, Li A, Wang Z and Han J: The advantages of using traditional Chinese medicine as an adjunctive therapy in the whole course of cancer treatment instead of only terminal stage of cancer. Biosci Trends 9: 16-34, 2015.

22. Chellian R, Pandy V and Mohamed Z: Pharmacology and toxicology of $\alpha$ - and $\beta$-Asarone: A review of preclinical evidence. Phytomedicine 32: 41-58, 2017.

23. Oprean R, Tamas M and Roman L: Comparison of GC-MS and TLC techniques for asarone isomers determination. J Pharm Biomed Anal 18: 227-234, 1998.

24. Huang C, Li WG, Zhang XB, Wang L, Xu TL, Wu D and Li Y: $\alpha$-Asarone from acorus gramineus alleviates epilepsy by modulating A-type GABA receptors. Neuropharmacology 65: 1-11, 2013 .

25. Yu X, Zhe Z, Tang B, Li S, Tang L, Wu Y, Chen X and Fang H: $\alpha$-Asarone suppresses the proliferation and migration of ASMCs through targeting the lncRNA-PVT1/miR-203a/E2F3 signal pathway in RSV-infected rats. Acta Biochim Biophys Sin (Shanghai) 49: 598-608, 2017.

26. Wang N, Wang H, Li L, Li Y and Zhang R: $\beta$-Asarone inhibits amyloid- $\beta$ by promoting autophagy in a cell model of Alzheimer's disease. Front Pharmacol 10: 1529, 2020.

27. Lee JY, Lee JY, Yun BS and Hwang BK: Antifungal activity of beta-asarone from rhizomes of acorus gramineus. J Agric Food Chem 52: 776-780, 2004.

28. Liu XC, Zhou LG, Liu ZL and Du SS: Identification of insecticidal constituents of the essential oil of acorus calamus rhizomes against Liposcelis bostrychophila badonnel. Molecules 18: 5684-5696, 2013.

29. Lee SH, Kim KY, Ryu SY, Yoon Y, Hahm DH, Kang SA, Cho SH, Lim JS, Moon EY, Yoon SR, et al: Asarone inhibits adipogenesis and stimulates lipolysis in 3T3-L1 adipocytes. Cell Mol Biol (Noisy-le-grand) 56 (Suppl): OL1215-OL1222, 2010.

30. Liu L, Wang J, Shi L, Zhang W, Du X, Wang Z and Zhang Y: $\beta$-Asarone induces senescence in colorectal cancer cells by inducing lamin B1 expression. Phytomedicine 20: 512-520, 2013. 
31. Wang JH: Traditional Chinese medicine and the positive correlation with homeostatic evolution of human being: Based on medical perspective. Chin J Integr Med 18: 629-634, 2012.

32. Meng MB, Wen QL, Cui YL, She B and Zhang RM: Meta-analysis: Traditional Chinese medicine for improving immune response in patients with unresectable hepatocellular carcinoma after transcatheter arterial chemoembolization. Explore (NY) 7: 37-43, 2011.

33. Cai Y, Xiong S, Zheng Y, Luo F, Jiang P and Chu Y: Trichosanthin enhances anti-tumor immune response in a murine Lewis lung cancer model by boosting the interaction between TSLC1 and CRTAM. Cell Mol Immunol 8: 359-367, 2011.

34. Chang MY and Shen YL: Linalool exhibits cytotoxic effects by activating antitumor immunity. Molecules 19: 6694-6706, 2014.

35. Hara M, Yuasa S, Shimoji K, Onizuka T, Hayashiji N, Ohno Y, Arai T, Hattori F, Kaneda R, Kimura K, et al: G-CSF influences mouse skeletal muscle development and regeneration by stimulating myoblast proliferation. J Exp Med 208: 715-727, 2011.
36. Rapoport AP, Abboud $\mathrm{CN}$ and DiPersio JF: Granulocytemacrophage colony-stimulating factor (GM-CSF) and granulocyte colony-stimulating factor (G-CSF): Receptor biology, signal transduction, and neutrophil activation. Blood Rev 6: 43-57, 1992.

37. Waugh DJ and Wilson C: The interleukin-8 pathway in cancer. Clin Cancer Res 14: 6735-6741, 2008.

38. De Larco JE, Wuertz BR and Furcht LT: The potential role of neutrophils in promoting the metastatic phenotype of tumors releasing interleukin-8. Clin Cancer Res 10: 4895-4900, 2004.

39. Gordon S: The macrophage: Past, present and future. Eur J Immunol 37 (Suppl 1): S9-S17, 2007

(C) $\Theta$ This work is licensed under a Creative Commons

Attribution-NonCommercial-NoDerivatives 4.0 International (CC BY-NC-ND 4.0) License. 